\title{
Las redes de apoyo en el contexto educativo escolar, familiar y comunitario: debate imprescindible para las prácticas educativas inclusivas
}

\section{The support nets in the school, family and community educational context: it debates indispensable for the inclusive educational practices}

\author{
PhD, Juana Bert Valdespino ${ }^{1}$ \\ jbertva@unae.edu.ec \\ Orcid: 0000-0001-8356-4853 \\ MSc, Irina Naranjo Bert ${ }^{2}$ \\ inaranjobert@gmail.com \\ Orcid: 0000-0002-9990-7854 \\ PhD, Alina Rodríguez Morales 3 \\ alina.rodriguezm@ug.edu.ec \\ Orcid: 0000-0003-12009-8802
}

Recibido: 1/4/2020; Aceptado: 1/6/2020

\begin{abstract}
RESUMEN
La formación del profesional de la educación, sobre todo la concerniente al campo de actuación de la enseñanza básica elemental, tiene retos esenciales dentro de su proceso de prácticas relativas a la inclusión. En este trabajo se aplicó una metodología didáctica con principios de la teoría grupal de aprendizaje, implicando la teoría psicológica que aporta conocimientos teóricos y metodológicos que rigen el comportamiento de los grupos humanos y su influencia sobre el individuo. El análisis lleva a las personas que por su vínculo directo representan un apoyo imprescindible para los niños con los que el estudiante trabaja desde su práctica y de los que necesita para incorporar modos de actuación loables en su formación.
\end{abstract}

Palabras clave: redes de apoyo, prácticas educativas inclusivas

\footnotetext{
${ }^{1}$ Universidad de Nacional de Educación del Ecuador, Azogues, Ecuador.

${ }^{2}$ Instituto Superior Tecnológico Sudamericano, Cuenca, Ecuador.

3 Universidad de Guayaquil, Guayas, Ecuador.
} 


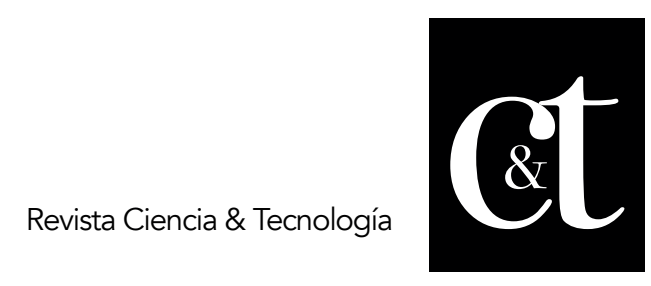

No. 27, 31 de julio de 2020

ISSN impreso: 1390 - 6321

ISSN online: 2661 - 6734

\begin{abstract}
The training of the education professional, especially that concerning the field of action of elementary basic education, has essential challenges within its process of practices related to inclusion. In this work, a didactic methodology was applied with principles of group learning theory, involving psychological theory which provides theoretical and methodological knowledge that governs the behavior of human groups and their influence on the individual. The analysis leads to the people who, due to their direct link, represent an essential support for the children with whom the student works from their practice and from whom they need to incorporate laudable modes of action in their training.
\end{abstract}

Keywords: support nets, inclusive educational practices

\title{
Introducción
}

Dentro de la enseñanza inclusiva el estudiante en formación tienen claridad que como parte de las políticas se aboga por cinco condiciones en las que hay que enfatizar a favor de estos estudiantes, ellas son: la cuestión de los derechos humanos; la igualdad de oportunidades y la anti caridad; las barreras sociales que llevan a la discriminación y la exclusión social; la consideración de estas personas como ciudadanos invisibles (son olvidados e ignorados, lo que se les impide tener una parte activa en la sociedad y la consideración de la discapacidad como parte de un grupo diverso.

Existe consenso de varias fuentes tratadas, que se ha de centrar la atención en la educación de estos niños como parte de la diversidad social, en la preparación de la comunidad educativa mediante el trabajo colaborativo para ofrecer una verdadera práctica inclusiva (Escudero, 2011), independientemente de las variabilidades del desarrollo. Se trata de ponderar el cómo y el con qué vías y recursos pueden insertarse a la sociedad, a partir de la preparación para la vida, no por tolerancia sino por aceptación, porque en esencia todos somos diferentes.

En este sentido se reflexiona en ese conjunto de personas que por su cercanía deben apoyar la inserción del niño y convertirse en la red social por la que las instituciones abogan, pues son consideradas parte ineludible del proceso de enseñanza aprendizaje, inserción y desarrollo de estos estudiantes.

Este trabajo aplicó una metodología didáctica con principios de la teoría grupal de aprendizaje, implicando la teoría psicológica que aporta conocimientos teóricos y metodológicos que rigen el comportamiento de los grupos humanos y su influencia sobre el individuo.

\section{Desarrollo}

Las redes sociales de apoyo se refieren a un conjunto flexible de personas con las que se mantiene un contacto frecuente y un vínculo social cercano. Estas personas son emocionalmente significativas y son quienes pueden brindarnos su ayuda, tanto de tipo material como emocional. Se basan en el trabajo colaborativo y pueden ser: la familia, los amigos, personal del colegio, vecinos, compañeros de trabajo, empleados de ciertas instituciones, y otras (Echeverría, 2016). 


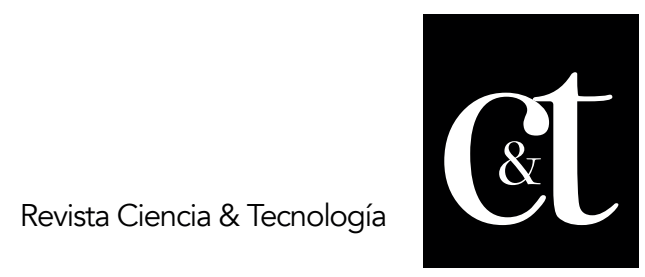

No. 27, 31 de julio de 2020

ISSN impreso: 1390 - 6321

ISSN online: 2661 - 6734

Monarca y Sandoval (2012, p.79) fundamentan que la colaboración, como característica esencial y necesaria de las prácticas sociales y educativas tiene un carácter fundamentalmente analítico, con la intención de favorecer su comprensión a partir del trabajo colaborativo de los profesores desde sus propias experiencias, las de estudiantes, padres o madres, $u$ otros profesionales. No es hasta finales del siglo XIX y durante el siglo XX cuando irrumpe con fuerza el sujeto como actor clave de los procesos sociales, a partir de entonces se ve que es necesario adaptar las circunstancias para que todos puedan desarrollar plenamente sus vidas en un mundo que es diverso.

Con el reconocimiento de la cultura de la diversidad, la UNESCO y los gobiernos de una gran variedad de países promueven prácticas educativas inclusivas y los docentes se encuentran en continua y permanente preparación para dar respuesta a este gran desafío.

Los fundamentos del trabajo colaborativo se relacionan con los cambios que se han producido y se producen a nivel social, las formas de vida, la tecnología, expresado en gran parte en la denominada sociedad del conocimiento. Pérez Gómez (1998, p. 163) confirma que la cultura docente "en una delicada encrucijada, viviendo una tensión inevitable y preocupante entre las exigencias de un contexto social móvil, cambian-te, flexible e incierto, caracterizado por la complejidad tecnológica".

Lo ético de toda práctica educativa, en tanto se vincula a un deber ser del sujeto y de la sociedad, y en cuanto siempre tendrá consecuencias, más o menos importantes, para el sujeto. En este sentido se dice que las prácticas educativas tienen un carácter normativo, porque reflejan, expresan y se orientan, hacia unas determinadas formas de entender al sujeto y a la sociedad. Por eso, impactan en la vida de los sujetos, de la comunidad y de la sociedad en la que viven.

Se produce una transformación del individualismo al trabajo colegiado, predominaba la institucionalización de la educación con la escuela y los sujetos vinculados a ella, la experiencia mediante el trabajo colaborativo y las evidencias que ofrece para las prácticas educativas inclusivas, que pasan de modelos homogenizados caracterizados por el trabajo individual a entornos y contextos heterogéneos donde todos aprenden diferente porque todos somos diferentes.

También, se analiza la desconexión existente entre las instancias de toma de decisiones sobre las intenciones educativas, el diseño del currículum, y las instancias donde este debe ser desarrollado. Desde el punto de partida, de los espacios que toman decisiones, muchas veces la educación es concebida como algo ajeno a los profesores, no se considera su participación y colaboración como profesionales en los proyectos y el currículo.

Desde las culturas colaborativas la escuela se considera una realidad construida y en construcción por complejos procesos democráticos, donde se piensa de manera colectiva; se comparte y debate en torno a los fracasos, dificultades e incertidumbres de forma abierta, con el fin de obtener ayuda, apoyo, consejo, y poder, así, mejorar y/o cambiar. Se fomentan, los puntos de vista alternativos; existen tiempos y espacios contemplados para el diálogo y la toma de decisiones de manera compartida. 


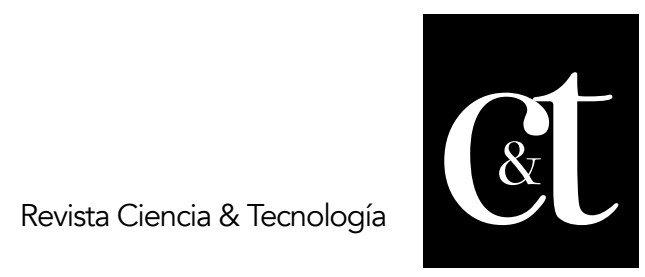

No. 27, 31 de julio de 2020

ISSN impreso: 1390 - 6321

ISSN online: 2661 - 6734

En estas escuelas se reconoce la voz, las intenciones, creencias y pensamientos del profesor, y demás miembros de la comunidad educativa. Se respeta su autonomía y profesionalidad. Se crean ambientes de trabajo satisfactorio y productivo, se promueve la colegialidad y el apoyo para el desarrollo profesional y aprenden y mejoran de forma conjunta.

Diversas investigaciones han demostrado la importancia de las redes sociales, las que están mediadas por la colaboración del otro en la vida diaria: situaciones de crisis, que precisan el apoyo proporcionado al encontrar soluciones, abrir nuevas posibilidades y disminuir la vulnerabilidad del individuo ante problemas físicos y emocionales. Por todo esto vale mucho la pena conocer quiénes forman parte de nuestras redes principales; así resulta más sencillo acudir a la persona indicada en el momento en que suceda alguna situación que pueda rebasar tu capacidad para afrontarla aisladamente.

Por ejemplo: en el contexto escolar, se emplean en las clases para colaborar con los amigos y al mismo tiempo realizar actividades que vayan de la mano con un estilo de vida saludable. Este hecho es coherente con los aportes de L.S. Vigotsky y sus seguidores (1995), cuando se aborda la importancia que tiene la creación de situaciones sociales del desarrollo a partir de un proceso de mediación social con los "otros". Se construye el aprendizaje mediante la colaboración con los otros con una participación colectiva basada en las vivencias y que tiene un condicionamiento histórico -cultural.

Otro ejemplo, en el ámbito del deporte y la promoción de salud, referido a cómo pueden ejercitarse en variadas actividades y horarios según su interés grupos representativos de personas, vivir la experiencia del trabajo en equipo y ponerte la camiseta de la Ibero, esta es tu mejor opción.

Son muchos los ejemplos que pueden ilustrarse como en la promoción cultural: actividades extracurriculares que promueven la generación de conocimiento y el desarrollo de las capacidades artísticas de la comunidad universitaria en su dimensión ético-estética, como elemento imprescindible de la formación integral: Artes Escénicas, Artes Visuales, Música y Literatura.

En la coordinación de organizaciones estudiantiles pueden conocer todas las actividades que sus compañeros estudiantes llevan a cabo en relación con su compromiso y varias causas humanitarias. Participar hasta donde las actividades escolares permiten, como: preocupación por los animales de la calle, por construir casas para la gente que ha perdido la suya, la lucha contra la delincuencia y la protección de los niños.

La participación como voluntarios universitarios en comunidades indígenas, en las actividades sobre medio ambiente, en la formación de promotores ambientales, agua, reciclaje, migración, refugiados, derechos humanos, género y feminismo, violencia contra la mujer, participación política y ciudadanía, acceso a la justicia, diálogo con representantes de las comunidades rurales, interculturalidad, pueblos indígenas y más opciones que coordinan los diferentes programas de incidencia. 


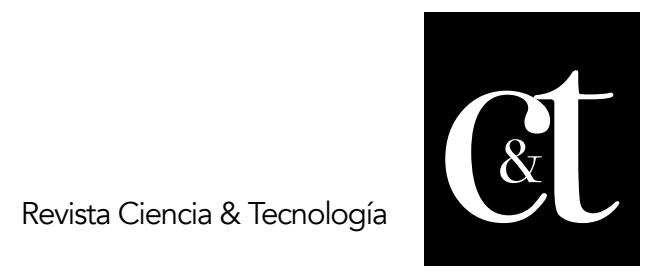

No. 27, 31 de julio de 2020

ISSN impreso: 1390 - 6321

ISSN online: 2661 - 6734

Un ejemplo más reciente lo constituye la labor de los voluntarios europeos para combatir el coronavirus en la distribución de alimentos y cuidado a los enfermos, independientemente de las restricciones por el contagio de la virosis respiratoria que surgió en China (diciembre de 2019) y se ha expandido por todo el mundo.

Las redes sociales y de apoyo se basan en el trabajo colaborativo y grupal, por las ventajas que proporciona para la solución de diversidad de situaciones educativas en diferentes contextos, que se fundamenta en un trabajo común, de ayuda y cooperación, basado en la solidaridad humana.

En el referido sitio consultado, se plantea que el trabajo colaborativo es aquel en el cual un grupo de personas intervienen aportando sus ideas y conocimientos con el objetivo de lograr una meta común. Se diferencia, sin embargo, del trabajo en equipo en que lo que se persigue en el trabajo colaborativo es la producción de conocimientos, y no tanto la optimización de resultados.

Estas ideas pueden ser discutidas porque el trabajo en equipo no solo es colaborativo por el apoyo del otro, sino que se construye el conocimiento con la participación de todos, con la inteligencia colectiva, todos pueden aprender desde una visión democrática y de respeto al otro. Sus dinámicas de trabajo, además, se diferencian fundamentalmente en que en el trabajo colaborativo figuras como la del líder o coordinador surgen espontáneamente, y no están rígidamente definidas. En este sentido, es una forma de trabajo más flexible que permite lograr resultados diferentes (no necesariamente mejores) que el trabajo en equipo.

Son ideas muy polémicas porque los fundamentos del trabajo en grupo han sido profundamente estudiados y de ninguna manera son rígidos, en tanto se negocia en la comunicación para que el grupo se sienta cómodo en la búsqueda de un propósito determinado. Por tanto, estas ideas son muy polémicas y no se comparten.

Ojalvo (2002), citando varios autores, señala que desde finales del siglo pasado la Didáctica viene insistiendo en la "enseñanza por equipos", el "plan de los grupos de estudio", "el trabajo en colaboración", la "comunidad de vida", las "comunidades escolares", la "enseñanza en grupos", el "trabajo por grupos", y otros métodos que apelan al aprendizaje colectivo con fines de educación social.

Estos autores precisan que el trabajo en grupo se inserta en las formas didácticas de estudio cooperativo que toman en cuenta la autoactividad y la formación de los sentimientos sociales, reuniendo a los alumnos en grupos reducidos para realizar las tareas asignadas por el profesor. De esta forma el énfasis está dirigido al rendimiento escolar, a la aplicación al estudio, a la autoactividad, a los hábitos de trabajo y cooperación.

El grupo es considerado como un lugar y medio de aprendizaje escolar, pero no se enfoca al grupo en sí como totalidad con un sentido propio. En la década del 30 el estudio del grupo como objeto de la Psicología Social y en especial la dinámica de grupo, entendida como cuerpo de conocimientos teóricos que permiten esclarecer los fenómenos grupales, adquiere un desarrollo crucial. Sin embargo, no es hasta inicios de la década del 50 que los educadores vuelven a encauzar sus esfuerzos en esta dirección; estancamiento que estuvo determinado entre otros factores por:

- La tendencia de la práctica educativa a exaltar al individuo y los métodos de enseñanza individualizada. 


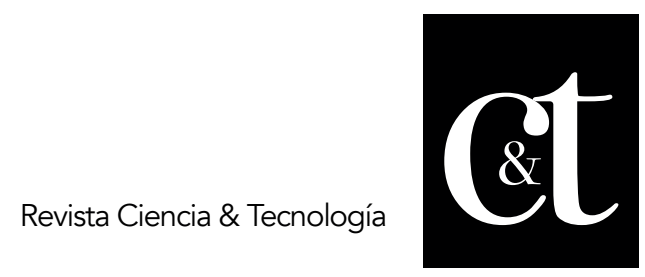

No. 27, 31 de julio de 2020

ISSN impreso: 1390 - 6321

ISSN online: 2661 - 6734

- La gran importancia otorgada por los educadores al uso de las técnicas de grupo, concediendo escasa atención a los principios subyacentes.

- La falta de exposición precisa de los conocimientos psicológicos más destacados sobre los grupos y de cómo pueden relacionarse con la metodología real de la clase.

Se precisa la elaboración de una metodología didáctica inspirada en los principios de la teoría de grupo y que se encuentra en la actualidad en activa elaboración y aplicación. A su vez, al aplicar una metodología grupal de aprendizaje, les resulta común la comprensión de la "clase" como grupo, y precisamente la teoría psicológica aporta conocimientos teóricos y metodológicos que rigen el comportamiento de los grupos humanos y su influencia sobre el individuo.

Desde la Psicología Social, el propio concepto de sociedad tiene un nivel de generalidad tal que nos obliga a precisar su alcance, y, sobre todo, la forma en que se presenta a los ojos del psicólogo.

Se definen como ámbitos principales de construcción y expresión de la subjetividad: el familiar, el grupal, el comunitario, el institucional y el social y como niveles psicológicos en que se conceptúa y visualiza la subjetividad en atención a principios teóricos y metodológicos enfocados a las particularidades genéricas de cada nivel: el personal, el interpersonal, el grupal y el masivo. Un alto valor en el orden metodológico tiene diferenciar, en el orden operativo, la relación diagnósticointervención.

La sociedad y el individuo son inseparables porque, en primer lugar, la inserción en la sociedad exige que el individuo posea una identidad que le permita entrar en determinadas relaciones sociales y una capacidad para abarcar estas relaciones y sus posibilidades. En segundo lugar, porque las condiciones de la sociedad penetran hasta el propio centro de la individualidad construyendo una subjetividad atravesada permanentemente por una pertenencia social particular.

Estos sistemas valorativos se concretizan en cada uno de los niveles de inserción del hombre en la sociedad, a saber: interpersonal, grupal, institucional y comunitario los que resultan ser, por lo tanto, niveles "productores" de subjetividad. El grupo entendido como un lugar de mediación y a la vez, como un privilegiado espacio de construcción y desarrollo de la subjetividad.

La fuerza y potencialidad del individuo, el grupo y la sociedad será aprehendida en su real dimensión si entendemos que constituyen tres polos de una relación dialéctica que les permite un permanente intercambio en y a través del cual se actualizarán las relaciones de influencia e interinfluencia que potencian.

La concepción de los apoyos se basa en diferentes perspectivas sobre la respuesta a las necesidades educativas de los alumnos según la propuesta de Ainscow (1995) y (Echeita y Simón, 2007). Desde la perspectiva individual o esencialista y la contextual o educativa. La primera, requiere ayuda especial, los apoyos se organizan para los alumnos que son considerados "especiales". El resto del alumnado permanece en los centros ordinarios, a los que no se presta ninguna ayuda extra al profesorado porque trabajan con alumnos "normales". La educación de este alumnado debe ser 


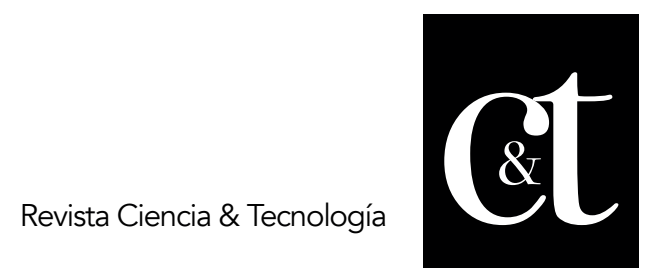

No. 27, 31 de julio de 2020

ISSN impreso: 1390 - 6321

ISSN online: 2661 - 6734

responsabilidad de un profesorado también "especial", en términos de conocimientos, destrezas y habilidades propias para trabajar con el mismo.

En la perspectiva contextual o educativa, cualquier alumno puede experimentar dificultades para aprender en un momento u otro de su escolarización. Los sistemas de ayuda y apoyo deben estar disponibles para todos los alumnos que lo precisen y se organizan a lo largo de su escolarización. Los profesores asumen la responsabilidad del progreso de todos los alumnos, con el compromiso de avanzar hacia una educación más inclusiva.

En esta red tienen que estar los alumnos y sus familias, los propios compañeros y la comunidad con todos los servicios relacionados con la atención a la infancia y la adolescencia, pero también con los recursos comunitarios que todas ellas, en mayor o menor grado, tienen (medios de transporte, empresas, comercios, museos, servicios públicos), se refuerzan los vínculos entre comunidad social, política y educativa (Parrilla, 2006).

Los distintos tipos de apoyos que pueden prestarse tienen una finalidad didáctica. Se mejora la participación, al sentido de comunidad y al fortalecimiento ético como parte del proceso de enseñanza-aprendizaje.

Se trata de percibir la escuela-red y los proyectos educativos, circunstancia en la que el centro de enseñanza como tal pasaría a ser nada más y nada menos que eso, el centro, el nodo central, movilizador, en vez del recinto exclusivo, de proyectos más ambiciosas articulados en redes más o menos amplias.

Echeita y Simón (2012) aluden a un marco de referencia para una visión ampliada del apoyo escolar, donde se relacionan los agentes con los ámbitos de actuación: mejorar la participación del sentido de la comunidad y del proceso de enseñanzaaprendizaje. Se refuerza el sentido de equipo, con apoyo moral y seguridad, que ejerce una influencia positiva. Permite una observación mutua, se crean espacios de docencia compartida, con apoyo directo y específico a los alumnos, se difunden experiencias y se facilita la gestión del aula.

En el alumnado se realiza la participación con la acogida de nuevos compañeros, se refuerzan las relaciones sociales: "círculo de amigos", los procesos de consulta, que mejoran el aprendizaje cooperativo, participan en las juntas de evaluación y aplicación de TIC.

En las familias; facilitan el sentido de comunidad, dan apoyo moral, confianza, estima y consideración, contribuyendo a la creación de redes de familias, participan en estructuras formales (consejo escolar), comisiones, aula, otras. Todo este proceso permite apoyar las actividades en los diferentes ámbitos de la vida de la escuela (aula y centro en general). La participación como voluntarios en "grupos interactivos", tanto desde aspectos prácticos a emocionales.

La participación de los servicios de la comunidad y los mediadores sociales, las asociaciones, las ONG, los grupos religiosos, la universidad y otros centros escolares. Se trata de una amplia red de participación para lograr prácticas educativas inclusivas.

Resulta interesante comentar la reflexión de los autores respecto a la tradición educativa poco o nada proclive a reconocer y respetar, en un marco inclusivo, la 


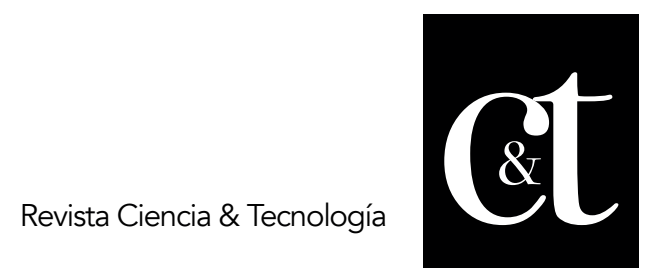

No. 27, 31 de julio de 2020

ISSN impreso: 1390 - 6321

ISSN online: 2661 - 6734

diversidad del alumnado. De ahí que sea estratégico capacitarse para implementar de forma continua y sostenida tales procesos de mejora e innovación, a partir de los apoyos existentes para aumentar y fortalecer la red que se necesita.

Es necesario asumir que el cambio institucional debe insertarse como un sistema integrado donde cultura escolar, política de centro y prácticas de aula se apoyan en mayor o menor medida. Desde una reflexión colectiva sobre aspectos o contenidos que condicionan el desarrollo y consolidación de la red de apoyo que se pretende.

Se ha de organizar y dinamizar adecuadamente estos procesos grupales para las sesiones de reflexión conjunta. El apoyo de asesores externos a modo de amigos críticos puede ser, en este punto, crucial. Los profesionales de la orientación educativa están en una posición envidiable para asumir este papel que, particularmente, es más positivo que otros que se realizan (Echeita y Simón, 2011).

Se ha de considerar las opiniones del alumnado y de las familias, a partir de cuidar las condiciones (momento, tiempo y lugar), con la voluntad de avanzar, buscar consenso de las prioridades con una perspectiva amplia sobre los apoyos. Debe tomarse como normal, en un proceso de cambio, la existencia de conflictos y discrepancias en el proceso de debate. La idea es mantener la dinámica de trabajo conjunto.

Para la implementación de esta concepción, el estudio retoma algunas publicaciones como el Índice para la inclusión (Booth y Ainscow, 2006), quienes utilizan instrumentos de gran valor y utilidad para guiar esta reflexión individual y colectiva, sobre las múltiples dimensiones y cuestiones que están sistémicamente relacionados con una red de apoyos que son útiles para esta finalidad.

El trabajo colaborativo en el contexto escolar se basa en el diálogo, la comunicación, la negociación y la explicación para estimular el aprendizaje, la interacción y cooperación social resulta más estimulante para el desarrollo del pensamiento. Está demostrado que la comunicación es la base de la educación (González, 1993). El diálogo permite contrastar puntos de vista y opiniones, la reflexión y el pensamiento crítico; el resultado de esa interacción es la adquisición de nuevos conocimientos.

Se reconoce que el trabajo colaborativo en el aula fomenta el sentimiento de solidaridad y respeto mutuo entre los alumnos. De ahí que el rol del docente es esencial, pues es quien debe implementar, coordinar y orientar el trabajo colaborativo en el salón de clases.

Existe una polémica con respecto al trabajo en grupo y el trabajo colaborativo y cooperativo. Por su parte, se aclara que el trabajo colaborativo es un trabajo en grupo; sin embargo, se cuestiona que este trabajo no es colaborativo. Se plantean enfoques diferentes: trabajo colaborativo y cooperativo. El proceso de enseñanzaaprendizaje se estructura mediante el trabajo colaborativo, la ayuda mutua, la cooperación y la solidaridad humana.

Se discute que el trabajo colaborativo es trabajo en grupo, pero el trabajo en grupo, no es trabajo colaborativo. Los enfoques de trabajo colaborativo y cooperativo, tienen algunas características que los diferencian notoriamente. Cada paradigma 


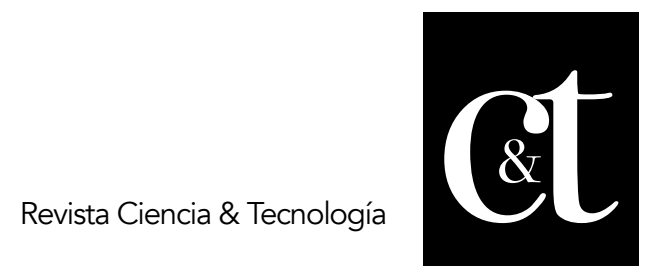

No. 27, 31 de julio de 2020

ISSN impreso: 1390 - 6321

ISSN online: 2661 - 6734

representa un extremo del proceso de enseñanza-aprendizaje que va de ser altamente estructurado por el profesor (cooperativo) hasta dejar la responsabilidad del aprendizaje principalmente en el estudiante (colaborativo). El enfoque colaborativo es el que requiere de una preparación más avanzada para trabajar con grupos de estudiantes (Monarca y Sandoval, 2012).

Desde los sustentos anteriores hay que reconocer que el trabajo en grupo requiere de un trabajo colaborativo y se basa en la cooperación del otro, tanto del profesor que guía, el alumno, los voluntarios o personal de apoyo, la familia, la comunidad, las organizaciones, en general la comunidad educativa que se involucra en el proceso de educación inclusiva.

\section{Conclusiones}

La educación en la actualidad requiere del trabajo en grupo. En las actividades de enseñanza-aprendizaje, el trabajo colaborativo o cooperativo (términos utilizados indistintamente) conforma uno de los principales elementos a tomar en cuenta.

Los proyectos innovadores que usan técnicas de enseñanza aprendizaje involucran esta modalidad de trabajo en la que el ser que aprende se forma como persona, pero en el que todos los actores participan desde sus diferentes competencias para el éxito de la educación inclusiva.

\section{Referencias bibliográficas}

Ainscow, M. (1995). Necesidades educativas especiales en el aula. Guía para la formación del profesorado. Madrid: Mad. S.L.

Booth, T. y Ainscow, M. (2002). Índice para la inclusión (folleto). Madrid: centro de Estudios para la Educación Inclusiva.

Echeita, G. y Simón, C. (2012). Cómo fomentar las redes naturales de apoyo en el marco de una escuela inclusiva. Madrid: Mad. S.L.

Echeverría, L. (2016). Redes de apoyo. Universidad Iberoamericana, México. http://estarbien.ibero.mx/mis-relaciones/amigos/redes-de-apoyo/, consultado el 15 de marzo de 2020.

Escudero, J. (2011). Educación inclusiva y cambio escolar. Revista Iberoamericana de Educación, 55: 85-105.

González Morales, L. (1993). Un Modelo de Comunicación Educativa en el aula a Nivel Superior. UNAM-ENEP Acatlán, Tesis de Licenciatura. México.

Monarca, H. y Sandoval, M. (2012). Profesores que se apoyan frente a la complejidad de la inclusión educativa. En cómo fomentar las redes naturales de apoyo en el marco de una escuela inclusiva. Madrid: Mad. S.L.

Parrilla, A. (2006). Grupos de apoyo entre docentes. Cuadernos de Pedagogía. Málaga: Aljibe.

Pérez Gómez, A.I. (1998). La cultura escolar en la sociedad neoliberal. Madrid: Morata.

Vigotsky, L. (1995). Fundamentos de Defectología. Obras completas, tomo V. La Habana: Pueblo y Educación. 